\title{
Braille reader
}

\section{- Old braille text for young -}

\author{
Yasuhiko Ogawa ${ }^{\dagger}, \quad$ Yoshinobu Kikuchi ${ }^{\dagger \dagger}, \quad$ Nobuyuki Ohtake ${ }^{\dagger}$ \\ $\dagger$ Tsukuba college of Technology, Japan. \\ †† Tokyo Kaseigakuin Tsukuba college, Japan.
}

\begin{abstract}
A braille reader is developed as an input device on a personal computer. This mechanism is direct touch for embossed point on braille paper by an isolated thin metal wire. We designed a braille reader considering standard input/output interface[3] of general computers, then this can connect any other large/small computers. Not only a braille reader is used by the blind and volunteer, but such a useful machine should be set up in public offices and libraries. Furthermore, this braille reader makes many copies of an old braille document which exists only one.
\end{abstract}

\section{Introduction}

When we think about education for the blind and social independence of a blind person, an input device of computer[1], which reads braille and tactile patterns, is increasingly required for (1) a regeneration or a reproduction of braille document or text, (2) a speech synthesizer, (3) a translation from braille text to some printed materials (reverse conversion), (4) a reading check for some important braille documents after writing, (5) a proof reading of tactile pattern and (6) an assistant/compensative equipment for someone who does not know about braille. Then these items are more and more necessary for the blind themselves and also an administration office of government or a related place from this time forth.

In an observation of braille information circulation, a writer records their documents to a floppy disk by using a personal computer or a word processor in recent years, and he uses this floppy disk as a distribution medium. Or someon: perhaps directly inputs a braille text or braille information (which contain $s$ tactile data) to a computer by using an on-line computer system. But all of the blind do not have a personal computer or any other machines. Then there are many cases that braille documents, which are written on a thick paper by hand using a braille board, are circulated for daily use generally. A braille hand writing tool (a board and a stylus set) is very convenient for carrying when we think about its weight, handling, no electric power supply and its size compare with a personal computer. Furthermore, a hand writing tool is extremely cheap 
in comparison with various kinds of other electronic information devices. Then such a hand writing tool is obviously utilized as a note taking tool of the blind from this time forth. In this fact, for example, there are some chances that the blind submit a document which is written by braille to a municipal office, a bank or any other reception offices. In other cases, some teachers may read a braille document written by students. In such cases, a small and simple braille reading device is necessary as a handy tool for a receipter who can not read braille. If such a device or machine exists, it is very easy to make many reproductions from an old braille document which remains only one in a library or personal collections.

\section{Development goal}

A braille reading device, which mechanism is an "optical method", has already been developed. For example, braille reproduction/copy device "Braille Master" is a famous commercial product. An optical method is as follows. When optical light from irradiated angle is applied to a braille sheet, the shade of embosses is fell upon the paper. These convex points or concave points of braille from oblique direction are read by an image scanner. A shortcoming of this method is against some dirt on the paper causes misreading. This is an unavoidable point in an optical method. Especially, old braille documents contain such weak points. In the case that braille is printed on both sides of the paper, each shade of the convex and concave points appears on one side at the same time. Very ad vanced processing is required to distinguish convex and concave points in such an optical approach. Currently it is very difficult to recognize the surface and reverse side braille points on one sheet by such optical method. A principle of this experimental reading device is that "convex points of braille are detected mechanically" at first, then analyzing braille or tactile figure by opening and closing a contact point of an electronic circuit[4].

We set up a development goal in the following manner.

1. A reader is not influenced by the dirt of braille sheet or tactile paper.

2. A reader is not influenced by the size of the braille character.

3. A reader can recognize weakly destroyed convex points.

4. A reader does not damage the original sheet and paper while the braille is reading.

5. A reader is not influenced by some inclination of the braille sheet.

6. A reader can correspond to A4 size, letter size and Japanese braille paper size.

7. Reading speed is at least less than 10 seconds for $\mathrm{A} 4$ size paper.

8. Small size and low price are required for personal use. 


\section{Mechanical principle and internal structure of a braille reader}

Our method is led by various experiments. A project point of braille is converted to mechanical movement of a touch sensor, this movement opens and closes an electric contact point. We judged that this way have high possibility of realization, because this mechanism is very simple. One physical touch sensor is made by a spring wire, this wire is bended to a right angle about 10 millimeters apart from the end (Figure 1 and Picture 1 show the touch sensor). This bended part is the mechanical contact point for a convex point of an embossed point and the opposite side of this wire is fixed. Bended wires guide a moving wire to a vertical position when it is rising up by a convex point. It can express equivalent circuit for one wire in Figure 1, positive logic output for the convex point is obtained from this.
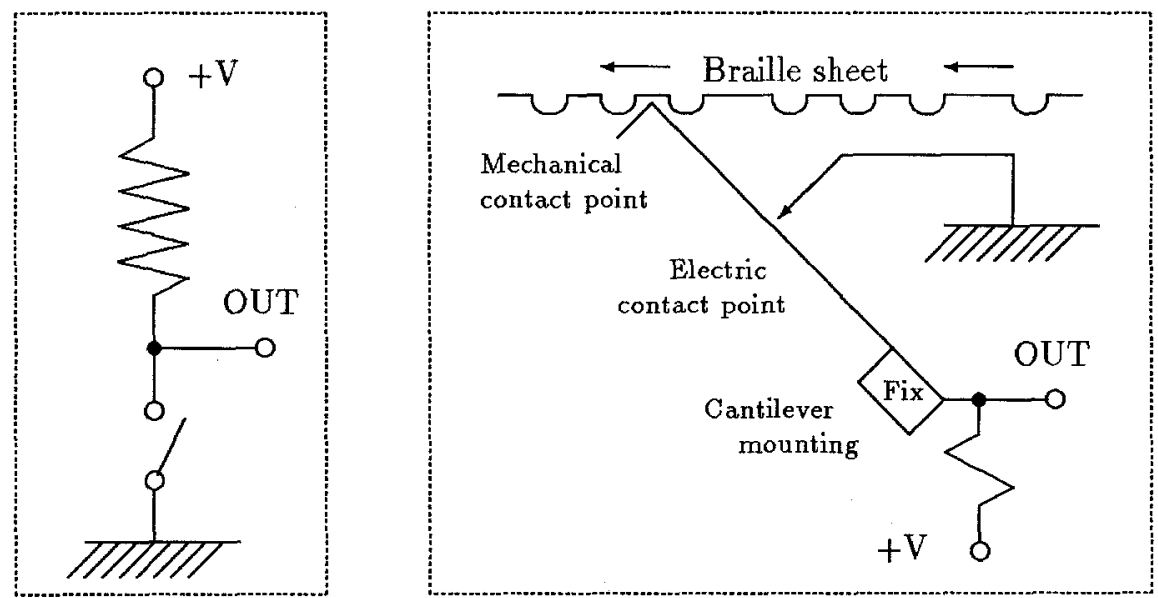

Figure 1. Positive logic output circuit \& its application.

Only concave points can be detected by changing a sensor position with the same mechanism. General demands from a user for standard devices are summerized in the following items, but detail requirements are not listed.

- Simple operation and high reliability for the machine.

- Easy trouble shooting.

Easy handling (it also includes pre/post process), the reliability and stability of a machine are essential elements. Then we materialized these items by the following methods in this research. (1) All controls for the braille reader are executed by a personal computer, then all operation switches are omitted from this device. If a braille manuscript is inserted to the reader, the sheet is detected, then a motor trigger works and the reading is started automatically. Even if the sheet reading finishes, the reader is in operating condition for a specified time and can read the next manuscript continuously. This operation time is variable, 
of course, if it does not insert the next manuscript within a specified time, the reader suspends. (2) There is high possibility of paper jamming during operation in such a machine that handles paper like a document copy machine. The sensor of the convex point, an electronic circuit relates to this sensor, a motor drive for input paper etc., these control functions are arranged inside the braille reader. When the paper is jammed, you can take it out easily by opening the top board in this design. (3) Pre/Post processing. We chose method that the manuscript is read with face down. The manuscript is stacked up on the discharge part after reading. The order of input sheets do not reverse, then a replacement of input sheets are not necessary for this operation. Thus, no complex operation is required for pre/post process.

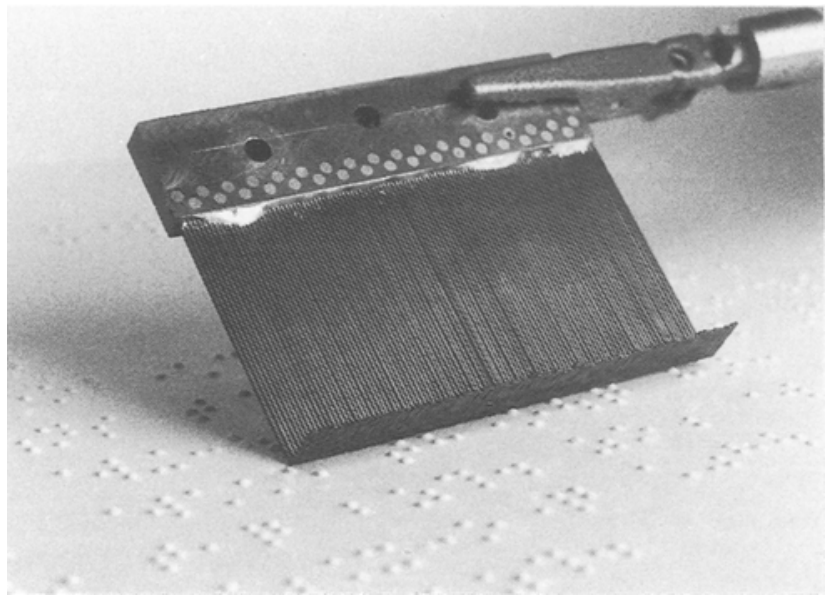

Picture 1. A sensor part.

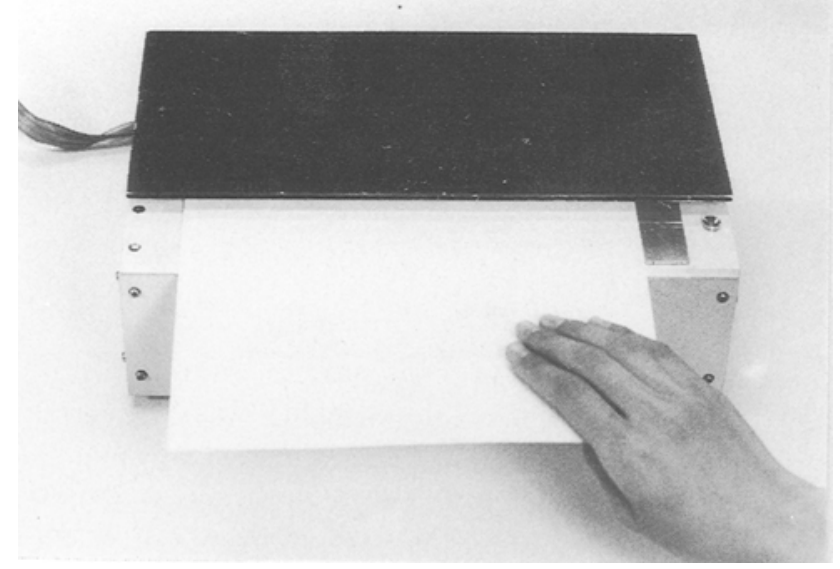

Picture 2. A braille reader. 


\section{Braille recognizing algorithm}

The next functions are necessary for automatic braille character recognization from an image data that is obtained from the braille reader.

- Detection of line position.

- Decision of braille character size.

- Pattern matching and skew processing.

In the decision process of line position, the image of braille points is projected in the horizontal direction at first, a line position is determined by searching the mode from the histogram. Vertical direction is the same as the horizontal way. Figure 2 indicates these horizontal and vertical histograms. Figure 3 shows outline of a character position decision way from these histograms.

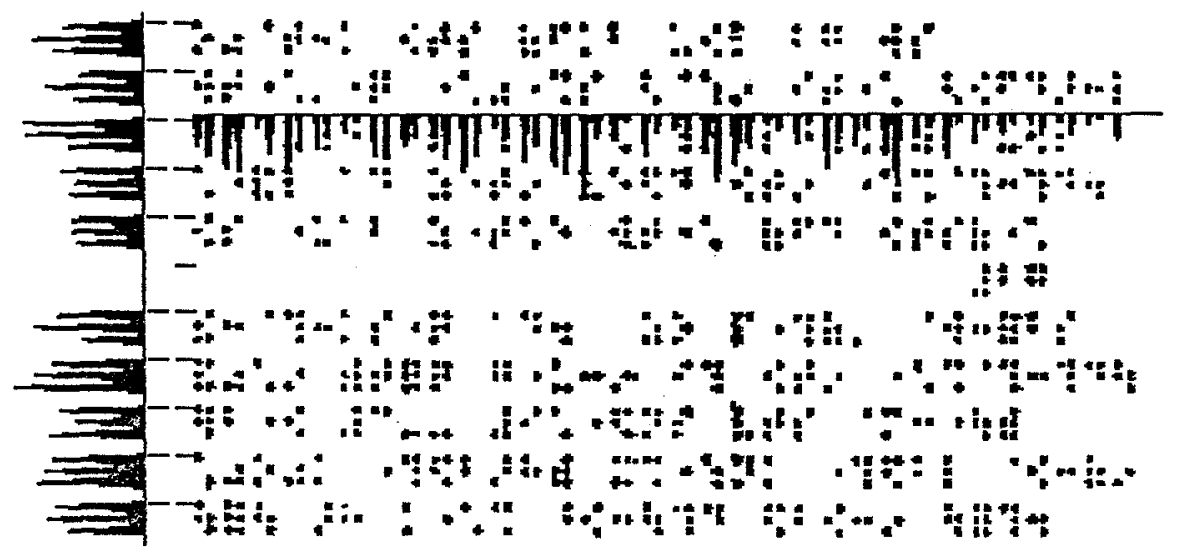

Figure 2. Horizontal \& Vertical histograms.

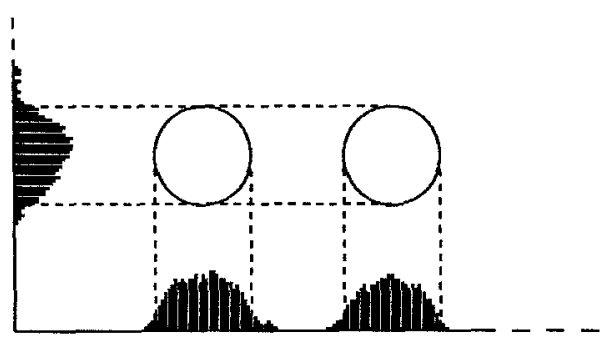

Figure 3. Logical hole.

The size of a braille character is obtained from the distribution of width direction. Both horizontal and vertical analysis in these histogram search methods, the distance between the braille letters and the braille character size can be decided antomatically. In the case that a histogram search area is defined the whole of the input sheet, when a piece of paper is inserted at an angle of 
some degrees for the touch sensor, it is very difficult to solve the mode from the histogram by this searching method. Because such a histogram has no good peak (data is flat). Accordingly, several lines that are at a specified length from the upper end of input sheet and some numbers that are at a designated length from the left end of the input, are the restricted target areas which are analyzed. Figure 4 shows relation between whole area and the defined region.
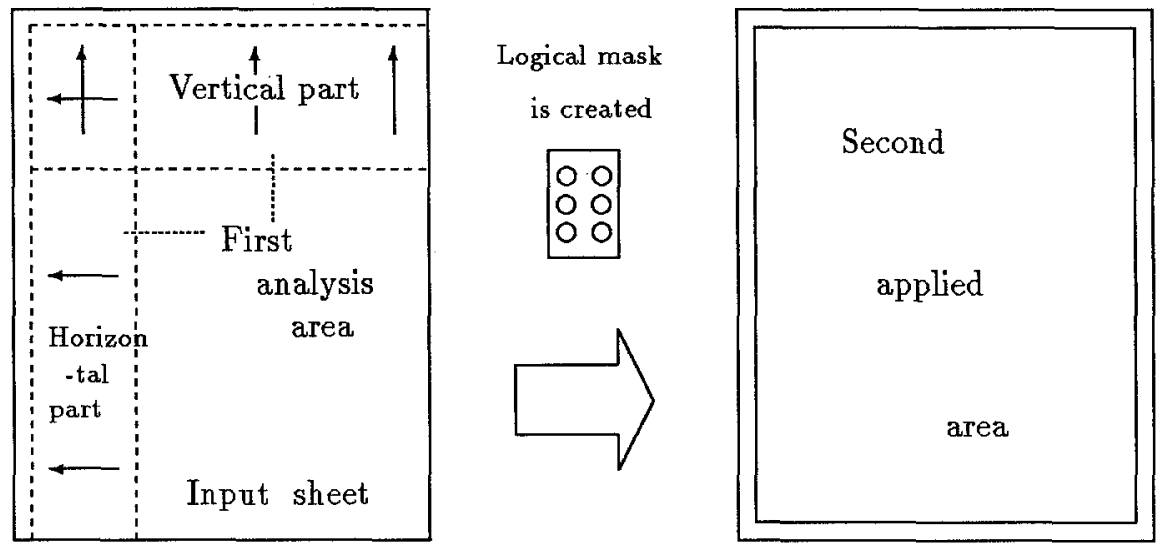

Figure 4. First analisys \& Second applied area in the input sheet.

This is a characteristic of this braille reader, thus decision of braille character size and a line space are treated statical research by restricted horizontal and vertical directions in this method. A statical analysis box area, which defines horizontal and vertical length, is justly variable. In the case that a numerical value which shows a page number does not appear in the histogram of vertical projection, because the page number is near the right edge of the braille sheet. Then a line position, which is placed in distant location, is determined to coordinate correction in estimation from horizontal analysis in this algorithm. At last a logical mask which has 6 holes is created from braille character size that is determined by this algorithm. At this time, the next three values have been defined from the restricted area.

- Distance between lines.

- Braille character size.

- A logical mask of 6 holes.

It should analyze the full area of the braille sheet as a second step by using this data and for a previous estimation. Overlapping this logical mask upon image data, it finds a good position which matches this logical pattern. If there exists some point which is bigger than regular value compared with the logical mask hole size, these points create a correspond braille code. This operation is iterated from left to right and top to bottom on full image data, and then this pattern matching finishes. In this pattern matching, this logical mask can move not only in a straight line but also in a wavey line, then it can analyze some inclined image data. 


\section{Actions}

A reader part consists of 430 sensors, these are arranged 2 sensors per millimeter. This dissolution ability is sufficient for small Japanese braille size compared with other countries' braille scale. Full image of the input sheet, which has braille and tactile patterns, is read by a moving the sheet, this minute transfer is a synchronized electronic pulse. Reading speed is 8 seconds for a A4 size sheet, when a MS-DOS personal computer (NEC : PC-98RL, CPU : Intel 80386, Clock : 20 $\mathrm{MHz}$ ) is used. It takes about 8 seconds to transform to braille and Kana ${ }^{1}$ letter (or English/number/symbol/Greek/Russian letter etc.,) from recognized image data. Recognized braille and Kana letters can be transformed from internal data to standard disk/floppy files, and print out directly to a braille printer and a normal printer respectively. And it is possible to read braille aloud into a machine by a combination of a voice synthesis device. When an irregular pattern is found in analyzed braille, it can add/delete one point of a 6 dot pattern by an editor which is contained in this control software. It is possible to add/delete/move one braille character, and modification of Kana letter is also easy by this editor. It can only express 64 kinds of character (contains space character) by 6 dots braille method, then digits, large/small English letter, Greek, Russian letter, etc., are needed several distinguished marks before individual words when the context is changed. There exists 8 dots braille method in Japan, but this is an unusual way. Although these marks switch a context, several previous marks are sometimes omitted in some mathematical expressions and computer program descriptions, thus humans can understand omitted marks from the context. But it is very difficult to recognize the hidden mark for this braille reader which is controled by software. When a previous mark is omitted, if a special description. (for example, chemical expression and it's symbol) is read as a general sentence, it will become a meaningless character set. Then we provided an interactive mode which can change the reading pattern. Braille patterns contained in tactile figure are recognized as braille characters by designating partially, and these characters are also transformed to print characters on display. This braille reader is a prototype product which reads braille and tactile figure patterns by arranging many mechanical sensors, this is the pursuit of its possibility and ability. Detailed contrivances are omitted in the first development step, therefore, only a flat sheet is passed though this sensor part continuonsly. This braille reader is weak at processing correctly a broken or distorted sheet. However the solution of this problem currently remains, materializing process has become clear. It will be scheduled about this weak point after this. Furthermore, recognition rate of this braille reader is $99.8 \%$ for a flat sheet which does not have a broken part or distortion.

\footnotetext{
1 "Kana" is Japanese phonetic alphabet.
} 


\section{Conclusion}

We proved "an input of braille and tactile pattern to a computer" by simply using a hand made touch type braille reader. It is very easy to convert from braille to Kana string, because Kana and Japanese braille have one to one correspondence like alphabet and its braille code. There is no difficult point in this Japanese conversion technically, then it can make braille reading software by voice synthesizer[2] without any problem. Although we developed an antomatic "Kana to Chinese character conversion"[6], this conversion program is currently an isolated software from this braille reader. Using this software, the blind can write a general document or print a text "by themselves" from braille to sentences mixed with Kana and Chinese character[5]. And it is also espected to convert from a tactile sheet to a visible print automatically. These are the applications of this braille reader in our research. Though there remained co-ordinate correction in estimation, smoothing method, and other problems (a treatment of Japanese, high performance, etc.), each problems are solved in visible prints and materials, an application for braille reading method is not difficult by improvement and combination of our technology and commercialized products.

\section{References}

[1] Margaret R. Barker, "Computer input devices for physically disabled people", IEEE, 26th COMPCON, 1983, pp.175-178.

[2] R.Marsden, L.Tanne and N.Durdle, "Computer-aided speech prosthesis", Proceedings of the SID, Vol.30, No.1, 1989, pp.45-49.

[3] Elliot Cole and Parto Dehdashti, "Interface design as a prosthesis for an individual with a brain injury", SIGCHI Bulletin, Vol.22, No.1, 1990, pp 28-32.

[4] Yasuhiko Ogawa, Yoshinobu Kikuchi, Nobuyuki Ohtake, et.al., "Technical report No.63880095" by a Grant-in-Aid for Scientific Research, Ministry of Education, Mar., 1990. (in Japanese)

[5] Nobuyuki Ohtake and Yuji Takano, "Japanese braille", The British Journal of Visual Impairment. Vol.10, No.3, Nov., 1992.

[6] Nobuyuki Ohtake, "Japanese braille translation system", The Journal of Visual Impairment and Blindness, 1994. (to be published)

$\begin{array}{lrl}\text { Yasuhiko Ogawa } & \dagger & \begin{array}{l}\text { Tsukuba college of Technology } \\ \text { Kasuga 4-12, Tsukuba, 305, Japan. } \\ \text { Yoshinobu Kikuchi }\end{array} \text { Tokyo Kaseigakuin Tsukuba college } \\ \text { Nobuyuki Ohtake } & \dagger & \begin{array}{l}\text { Azuma 3-1, Tsukuba, 305, Japan. } \\ \text { Tsukuba college of Technology } \\ \text { Kasuga 4-12, Tsukuba, 305, Japan. }\end{array}\end{array}$

